\title{
Pediatric Hospital Medicine Core Competencies
}

\author{
Section Two: Core Skills
}




\section{BLADDER CATHETERIZATION/SUPRAPUBIC BLADDER TAP}

\section{INTRODUCTION}

Bladder catheterization is a common procedure, typically used to collect a sterile urine sample for analysis and culture when urinary tract infection is suspected. Bladder catheterization is also used to relieve urinary retention or obstruction, particularly in cases of anatomic abnormalities or neurogenic bladder, or to monitor urine output and fluid status. Pediatric hospitalists frequently encounter patients requiring bladder catheterization and should be adept at performing this procedure in infants, children, and adolescents.

\section{KNOWLEDGE}

Pediatric hospitalists should be able to:

- List the indications and contraindications for bladder catheterization.

- Describe how the method used to collect a urine specimen can affect interpretation of urine culture results, and explain why bladder catheterization or suprapubic tap are the preferred methods of collection in infants and children that cannot reliably produce a voided specimen.

- Review the basic anatomy of the male and female genitourinary tract.

- Review the steps in performing bladder catheterization for both male and female patients, attending to aspects such as patient identification, sterile technique, positioning, equipment needs, and specimen handling.

- Discuss the indications for analgesia, sedation, or anxiolysis and describe the medications that may be used for each.

- Describe the risks and complications associated with bladder catheterization, such as localized trauma, creation of a false passage, and potential stricture formation.

- List the indications for consultation with a urologist with regard to bladder catheterization, including known genitourinary tract abnormality, recent genitourinary surgery, or urethral trauma

- Compare and contrast the effects of using various methods to collect a urine specimen, including interpretation of the culture and urinalysis and patient risk,

- Define a UTI as obtained by various methods such as catheterization, clean catch, clean bag, and suprapubic tap.

- Discuss the importance of appropriate specimen handling and the effect on culture results.

\section{SKILLS}

Pediatric hospitalists should be able to:

- Perform a pre-procedural evaluation to determine risks and benefits of bladder catheterization.

- Demonstrate proficiency in performance of bladder catheterization on infants, children, and adolescents.

- Consider the level of pain and anxiety provoked by the procedure and provide appropriate pharmacologic or non-pharmacologic interventions when indicated.

- Correctly identify the need for and efficiently offer education to healthcare providers on proper techniques for holding and calming patients before, during, and after bladder catheterization.

- Consistently adhere to infection control practices.

- Identify complications and respond with appropriate actions.

- Identify the need for and efficiently access appropriate consultants and support services for assistance with analgesia, sedation, anxiolysis, and performance of a bladder catheterization.

\section{ATTITUDES}

Hospital physicians should be able to:

- Recognize the importance of obtaining a sterile urine specimen in order to correctly diagnose urinary tract infection.

- Communicate effectively with patients and the family/caregiver regarding the indications for, risks, benefits, and steps of bladder catheterization.

- Role model and advocate for strict adherence to infection control practices 


\section{SYSTEMS ORGANIZATION AND IMPROVEMENT}

In order to improve efficiency and quality within their organizations, pediatric hospitalists should:

- Lead, coordinate or participate in the development and implementation of cost-effective, safe, evidence-based procedures and policies for performance of bladder catheterization in children.

- Lead, coordinate or participate in the development and implementation of educational initiatives designed to teach the proper technique for bladder catheterization to learners and other healthcare providers. 